\title{
RE-THINKING THE METAPHOR OF SHADOW: FROM PLATO TO SHAKESPEARE AND ZEFFIRRELLI
}

\begin{abstract}
In any work of art, from literary to visual, theatrical and cinematographic, the presence of shadow, most of times associated with the presence of light, emerges as a particularly motivated sign. The very act of placing a shadow in a specific context makes the shadow subject to the same area of interpretation as the rest of the artwork. Within each artistic field, there are lots of meanings associated to shadow: from philosophical, theological and psychological to symbolic and metaphorical. According to the artistic medium it belongs to, the metaphor of shadow is usually engaged in creating and modifying the atmosphere, in visualising images, in diminishing or increasing the dramatic intensity and mainly in accumulating meaning. The present study intends to explore the transposition of the metaphor of shadow from its allegorical roots, as in Plato's allegory of the cave, into new artistic environments such as literary and cinematographic. Be it employed in the service of an idea, as in Shakespeare's Hamlet or used for creating a visual and cinematic effect as in Zeffirelli's homonymous film, the metaphor of shadow adapted its means of expression in order to extend the area of significances.
\end{abstract}

Keywords: shadow; metaphor; significance; literature; cinematography.

\section{The Metaphor of Shadow and Its Philosophical Roots}

Before focusing on the manner in which the metaphor of shadow functions in different artistic media it is necessary to make a few remarks regarding its philosophical roots. Transposed from a literary into a visual-spatial background, the metaphor of shadow becomes engaged into the process of modifying the whole atmosphere, of increasing or diminishing the dramatic intensity, of accumulating meaning that may further generate new effects for each time it is used in a cinematographic representation of a literary fragment.

From a wider philosophical perspective, the metaphor of shadow could be

* The National University of Music, Bucharest; Romania. 
identified as the central metaphor of Plato's cave allegory. ${ }^{87}$ Employed for stating Plato's theory according to which the world that the man is facing is nothing but a dim representation of the real world, meaning the world of ideas, the cave allegory introduces shadow as a philosophical metaphor. Thus, for the persons living in a cavernous cell down under the ground, enchained and having their heads fixed so that they could only look straight ahead to the walls of the cave, the shadows they watch, representing, in fact, other persons moving behind them, somewhere between the firelight and the cave walls, may appear as the actual reality. (Plato 240) The only person that was freed managed to escape the cave and to get outside into the sunlight where he started to experience the meanings of the new world. He became able to perceive certain aspects of the enlightened objects or persons as real, in the true meaning of the term. Although he returned to the cave in order to tell the others about the real world, no one believed him, considering that it was not even worth trying to get out. (243)

The allegory of the cave was employed mainly for depicting the relationship established between reality and its various shades and, at the same time, between reality and what is believed to be the real world. Although the shadows of the persons moving behind the prisoners are regarded as visual representations of the carriers, they are not perceived as shadows of something since the prisoners do not have any relation to light as light, since they do not see the fire that casts the light. (Heidegger 20) From this point of view, for the prisoners, all the shadows they perceive represent reality and not representations of reality ${ }^{88}$ except the freed prisoner for whom the shadows stand for reduced representations of actual persons. (Plato 242)

In the case of cave allegory, the perception of reality is made empirically, but the senses alone proved unable to provide true knowledge of the actual world. That is why, considering Plato's philosophical perspective, the empirical world of senses is somehow an imitation of the shadows of the highest reality, the reality of pure ideas. The process of perceiving the true reality turns to be a journey of the mind ascending into the intelligible realm of thoughts and ideas. In other words, the highest reality is, the most ideal it becomes.

When employed in different artistic media, the shadow receives new

\footnotetext{
87 The text is included in Plato's Republic Book VII which was written as a dialogue between Galucon, Plato's brother, and Socrates, his mentor.

88 This explains why the prisoners, having access only to a reality of shadows, did not believed the freed prisoner who tried to reveal the actual reality to them.
} 
specific features and connotations that highlight the means of artistic expression and representation. The meanings associated with the metaphor of shadow confer many new analytical interpretations to the works of art that make use of it. Having multiple rhetorical and symbolical valences, throughout time, the metaphor of shadow was ingrained in language and discourse as well as in art and culture. From the literary field ${ }^{89}$ that registers the use of shadow in tales, myths, legends, allegories and lots of narratives, to the field of art ${ }^{90}$ that exploits the representational possibilities of engaging shadow in such a way that it may generate further meanings on the symbolic and psychological level, the metaphor of shadow adapted its means of expression so that the area of significances could be extended. (Stoichiță 128-30) The aim of the present study is to explore the transposition of the metaphor of shadow from literature to cinematography focusing on the ways the employment of this metaphor becomes able to produce new meanings.

\section{The Metaphor of Shadow - From Literature to Cinematography}

The connection between the two artistic fields, literature and cinematography, is delivered by Shakespeare's Hamlet. For both cases, the metaphor of shadow was engaged according to the specific means of representation. As a powerful trope, this metaphor played an important part for the literary as well as for the cinematographic expression. Influenced by the philosophical perspective, the figure of shadow could be identified as an entity that seems to derive its identity from another entity, which is distinct from it. From this point of view, the figure of shadow may function as a basis for the evolvement of the relationship established between substance and appearance, between presence and absence and so on. Because of its metaphorical status, the shadow is not only able to interconnect these binaries but it can also animate the physical absence of a character into its ghost or it can embody spirits that are no longer able to speak up, or it can modify space so that it reveals the emotional

\footnotetext{
${ }^{89}$ The employment of the metaphor of shadow is very numerous in the literary domain, covering all literary genres, only to mention Dante's Inferno inhabited by the shadows of the lost souls, or Von Chamisso's Peter Schlemihls' soul symbolized by the shadow that was cut off and sold to devil.

${ }^{90}$ Beginning with the period of Renaissance, the visual representation of shadow started to be essential to the simulation of shape, perspective and relative depth and it changed the manner of painting. (Alberti 30-2) Thus, the painting became a product of empirical manipulation of the viewer's visual perception.
} 
experiences faced by a character.

Making use of its specific instruments, the literary field proposes various ways of engaging shadow in a metaphorical manner. In the first instance, it is the ghost, a character impersonating Hamlet's father claiming the revenge of his death, a shadow-like figure that seems real to those who see it. Then, there are the actors performing a play within a play, functioning as shadows in the role of shadows depicted from reality. ${ }^{91}$ In both cases, the characters impersonating ghosts are demanding remembrance as well as action or reaction from the living. At the same time, the prolonged hesitation to act under the influence of ghosts' direct or indirect influence may suggest that these figures also need to confront their witnesses with the possibility that the so called ghostly encounters could be regarded as dreams.

Besides the use of shadows as figures that are connected to the threat of mortality, the engagement of shadow was also preferred for a more rhetorical manner of literary expression. Feigning madness, Hamlet insists on the fact that he suffers from bad dreams which help him refer to the dissolution of any limit between the world of the dead and the world of the sleeping. The same concept of dream was related to ambition which, in its turn, makes a distinction between powerful figures, such as heroes, and beggars and makes Hamlet state that one is nothing but a shadow of the other.

HAMLET: there is nothing either good or bad but thinking makes it

So. To me [Denmark] is a prison.

ROSENCRANTZ: Why, then your ambition makes it one; 'tis too narrow for Your mind.

HAMLET: O God, I could be bounded in a nutshell and count myself a King of infinite space, were it not that I have bad dreams.

GUILDENSTERN: Which dreams indeed are ambition; for the very substance

Of the ambitious is merely the shadow of a dream.

HAMLET: A dram itself is but a shadow.

ROSENCRANTZ: Truly, and I hold ambition of so airy and light a quality that

It is but a shadow's shadow.

\footnotetext{
${ }^{91}$ By portraying the shadows of the true victims, the actors reinforce the power of meta-drama.
} 
University of Bucharest Review $\approx$ Vol. X/2020, no. 2 (new series)

Re-writing, Re-imagining the Past (II)

HAMLET: Then are our beggars bodies, and our monarchs and

Outstretched heroes the beggars' shadows. (Hamlet 2.2.244-57)

Based on literary art, the association of shadow with the supernatural, with the world of the dead, generates many possibilities of creating illusions, of manipulating not only the plot but also the characters' actions and/or reactions. Even more, shadow may convey a metaphorical aspect to concepts like madness, death, guilt and others. At the same time, shadow may function as a rhetorical device engaged in the process of modifying the space that comprises the known world as well as the other world beyond it, of increasing and/or diminishing the dramatic intensity, of constructing meaning.

Transposed into a cinematic medium, the shadow adjusts its means of representation so that they become fit to express further meanings. Most of times, the shadow is inserted in a cinematic frame as a companion to its caster, being perceived as a natural phenomenon unable to function on its own, but in this case, shadow not only reveals important aspects concerning the caster it is associated to, but it also builds up new important meanings. Regarded as a rhetorical and metaphorical device, shadow becomes able to convey meaning on the basis of the principles of visual perception and reception. As a representative form in its own, the shadow can offer many expressive possibilities in order to construct persuasive imagery that aims to modify the whole area of significances. ${ }^{92}$ As any rhetorical instrument, the shadow can be used as an instrument of persuasion, capable to modify the viewer's attitude. Far from being just a decorative device, the shadow may be employed in such a way that it can deviate the audience's expectations from what is considered natural in a cinematic frame. Being inserted in a certain frame, the shadow redesigns the meaning of the whole content.

From a wider point of view, it is already known that the human visual system usually perceived shadow unambiguously anchored to its caster either real or extracted from images. (Dee \& Santos 3-4) Any occurrence of a shadow in a certain image provides specific information concerning the casting object, the light source and the surface. All these items of information create the basic conditions that account for any viewer's expectations for the perceived shadow

92 From a combined rhetorical and metaphorical perspective, the perception as well as the interpretation of the use of shadow in a cinematic medium, are guided by the basic rules of the same principles that usually guide the perception and the understanding of the natural shadow. 
to be unambiguously anchored to the casted object. When engaged in a cinematographic medium, the use of shadow may be altered or modified in such a way that it may correspond to the new artistic or aesthetic intentions. Thus, the so-called anchoring rules are violated generating assumed incongruities between the audience's visual expectations and the actual visual representation of a specific frame or scene. In order to be taken into consideration as rhetorical and metaphorical device of meaning construction, any act of such violation that is related to the use of shadow, needs to meet the principle of relevance. Such a principle states that the viewer needs to search for meaning in any provided signal, assuming the fact that the signal is meant to support all the communicative intentions that are relevant to him. As a consequence, even the aforementioned incongruities are to be appreciated for their own message and, thus, generating the possibility of interpretation by depicting relevant meaning from the incongruities' presence in a certain cinematic context. (Forceville 84-86) For serving the metaphorical purposes, the use of shadow should lead the viewer's perception to these incongruities that, in order to generate further meaning or to modify the present one, have to violate, at least, one or few anchoring rules. In addition, they also have to be assumed as deliberative and assimilated according to the principle of relevance. Moreover, they have to relate the meaning identified in such incongruities to the artistic and communicative intentions.

\section{The revealing shadow}

The first type of shadow incongruities involves the use of shadow in such a way that it could manipulate the visual perception of the frame. By violating the anchoring rule of the required resemblance between the caster and its shadow, the incongruity that relates the two entities may distort the shape of the shadow so that it no longer represents the caster and becomes an entirely distinct entity. Even more, for more dramatic effect, the shadow may be replaced by another distinct entity suggesting the possibility of a common identity between the caster and its shadow. ${ }^{93}$ In this case, the incongruity focuses on transcending the physical connection between the two entities to the conceptual level. In spite the

93 The use of shadow as a visual representation of its caster's identity could be identified since ancient times. The very first profiles of human's shadow were traced by simple lines on the walls, setting a conceptual connection among the caster, its shadow and the visual representation. (Casati 156-7) 
fact that the two elements are known as dissimilar, the incongruity established by the use of the revealing shadow becomes capable to suggest the possibility of them to be conceptually identical. Thus, the shadow is revealing hidden aspects of its caster while the caster is showing what the message seems to be.

The whole ensemble comprising the caster and its shadow is positioned in the middle of the frame so that the centre of visual importance may receive the major interest. It is lit by a light source located outside the field of vision focusing only in the caster's face. The shadow is projected on the walls on the back and replaced by another entity as seen in figure 1 . By juxtaposing the two entities in a single frame it may be suggested that they are able to establish metaphorical relations between them. However, the two entities are distinct from each other and despite the visual resemblance one cannot substitute the other. A closer look

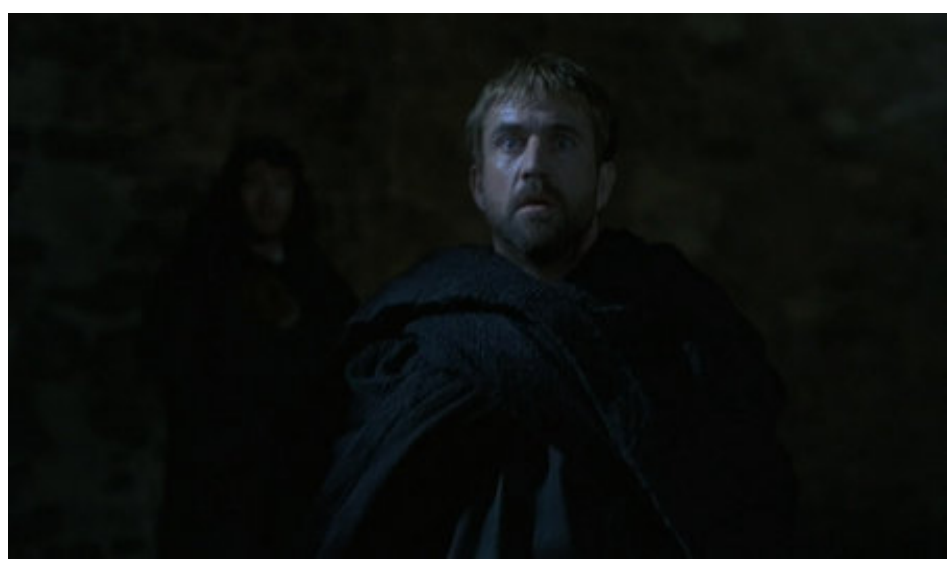
at the identity relation may suggest a difference in status and character's purpose. ${ }^{94}$

Since the connection between the two entities is developed on a metaphorical ground, it is possible for one of the entities to be taken as the metaphor target and the other as its source entity. (Lakoff \& Johnson 106-108). The presence of an entity in the position where the caster's shadow is expected to be encountered generates the assumption that each shadow may impersonate other entities, each shadow may be regarded as a person, just like the shadow of the ghost bringing to life Hamlet's father. Knowing that metaphors also serve to describe facts about the real universe, they can further connect real entities to entities from the world beyond, as if belonging to the real world. (Walton 293)

Similar to the use of shadow in Plato's cave allegory, the revealing shadow is regarded as being the caster. By positioning a second different entity in the

\footnotetext{
${ }^{94}$ In this case, the entity of the caster is facing the ghost, having the status of an actor, while the entity of the shadow is observing the scene, exhibiting the status of an observer.
} 
place where the caster's shadow is usually found, the notion of the shadow was extended and thus the shadow could be interpreted as being the caster's double revealing further features concerning the caster. (Casati 8-9) Playing the part of an observer, the revealing shadow is also rendering another aspect of the caster's identity, meaning the observer. ${ }^{95}$ In other words, Hamlet's double role, actor and observer, may be metaphorically suggested by the insertion of the revealing shadow in order to make the character's status as well as the aim of his future actions more explicit. Despite the fact that the caster and the shadow represent distinct entities, the incongruity between the two can be solved, on a conceptual level, by considering the revealing shadow to be the caster's possible alter-ego or other-self. (Stoichiță 136-138)

\section{The transitional shadow}

The use of the second type of shadow, like the first one, violates the same anchoring rule of the required resemblance between caster and its shadow. The incongruities developed between them are based on the fact that caster and shadow are, in fact, representing the same entity but, at the same time, they are able to manipulate the use of shadow in such a manner that it can capture the casting entity in different stages, such as attitudes, poses or qualities. The engagement of this type of shadow involves a transition ${ }^{96}$ from one position to another or from one attitude to another. The topic that connects the two stages needs to be a factor that causes the relation

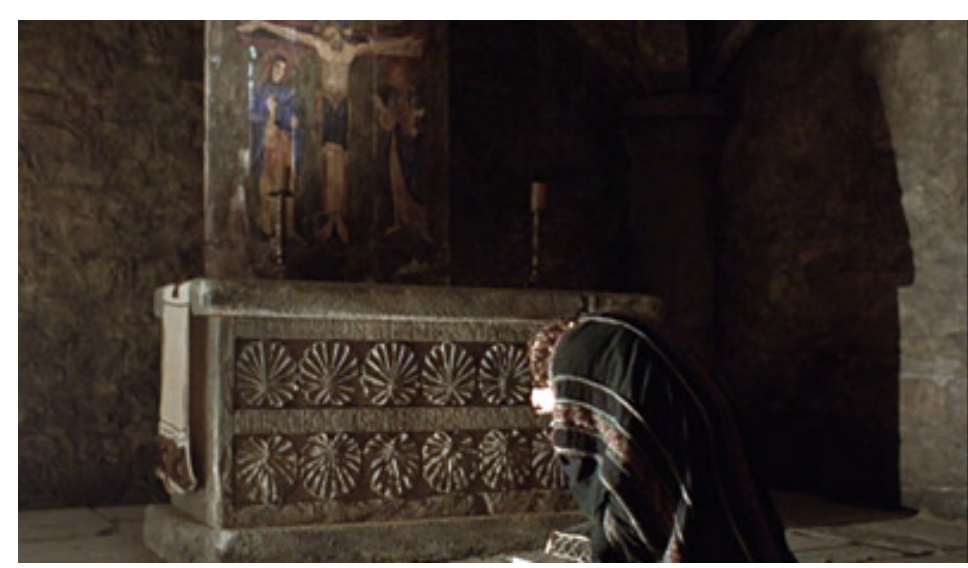
between them.

The employment of transitional shadow definitely involves a temporal evolution limited by the two border stages. Based on before-after transition, the stages can be related to a character's attitude, position, mimic, gesture and so on. Moreover, they could be extended to different actions or contexts. However,

${ }^{95}$ Additionally, the employment of the revealing shadow sets up the context for the metaphorical characteristics of this type of shadow to be exploited as visual make-believe cinematic instruments.

${ }^{96}$ From this point of view, the transitional shadow is more dynamic than the first type. 
what mainly characterizes the incongruities established by the employment of this type of shadow is the necessity of building up a relation of transition between the two stages of an entity evolution, stages which include both, the caster and the shadow.

Considering Claudius as playing the caster's part, the transitional shadow is put in relation to his inner states as haunted by guilt. While entering the space of praying, Claudius' silhouette is standing tall and the shadow is reduced to a small line covering the right column in the corner. The source of light comes from outside and not from the candle as seen in the second figure.

During his pray, Claudius is bending forwards and thus his body gradually becomes smaller while the shadow from the corner is more and more extended till it covers him, obtaining a transition in motion. In this case, the transition moves from caster to shadow. For creating a more dramatic effect, the angle of shooting was moved from the viewer's position to the warm's perspective enlarging the visual importance of the shadow. The causal topic ${ }^{97}$ that sets the transition is the guilt that is emotionally experienced by the character.

Similar to the use of first type of shadow, the employment of the second type also exhibits visual representation, meaning the character's silhouette and the shadow, but this time, the transition between the two border stages that reveals the relation established between them is governed by an emotional state. It is Claudius' sorrow that makes the shadow grow larger, stretching in an attempt to hold on to its caster. On a conceptual level, the dark shades of guilt and sorrow concern the shadow that represents the character's inner state.

In a true cinematic manner, the shadow can be manipulated so that it may capture the casting entity revealing not only his physical positions as gradually evolves form one stage to another but also his emotional experiences as unveiled in the same process.

\section{The indexical shadow}

The employment of this third type of shadow leads to the possibility of shadow to function as an index for the incongruity developed between the two entities. Different from the first two cases, the locus of incongruity is represented either by the caster or by the light source. In order to extend the shadow's

\footnotetext{
${ }^{97}$ Most of times, the topic identified in the incongruities delivered by the use of transitional shadow represents the factor that causes the suggested transition.
} 
properties over the constituents, meaning the caster entity and the source of light, it is necessary for the generic anchoring rule to be violated. In doing so, the features of the incongruent entity are also brought about by the other entities covered by the indexical shadow.

The use of shadow as an index aims to signal that one of the constituents represents an incongruity. Therefore, the incongruity concerns only one of the entities. Considering Peirce's perspective on the theory of signs, it is well known that the index establishes a dyadic relation. In Peirce's terminology, an index is a sign that refers to the object it denotes by virtue of being really affected by that object. By denoting the object, the index ${ }^{98}$ is drawing attention to the object with which it reacts. From this perspective, the index is meant to place the interpreter into mediate reaction with that object. (Peirce 306) Starting from this point, the concept of a sign could be extended beyond the traditional paradigm of language towards other fields. In the case of cinematography the benefits are numerous. Since an ordinary photograph ${ }^{99}$ of a particular individual could be taken as an index, the whole film can be regarded as a medium proper for iconic cinematic representation, as in animation, or indexical cinematic representation, as in photographic.

The indexical relation developed between the caster and the source of light uses a depiction of a shadow that emphasizes the incongruity of one of these two elements. The shadow becomes as important as the light drawing more attention to one entity.

As it can be noticed in figure 3 (The indexical shadow), the source of light is situated outside the area of vision, focusing on Gertrude. Her face is depicted from the general shadow that covers the room in such a way that the viewer's attention is led towards it, as if it is the most important spot of the frame. The entity that interrupts the focalization is Hamlet, perceived as a source of shadow, casting his shadow on Gertrude's right side. In this case the incongruity results from the unexpected slight projection of the shadow.

As if having its own shadow, this time coming from within, the entity is

${ }_{98}$ For example, pronouns denoting existent objects may function as indices within a language. Moreover, any type of gestures could be regarded as indices as well.

${ }^{99}$ A photograph may correspond to its subject based on a physical connection brought about by the effect of light reflectance from the subject to the camera. The resemblance between the subject and its photographic representation is due to the fact that the photograph was produced under specific circumstances that the two entities were forced to correspond completely. (Wollen, 84-6) 
unable to cover the other entity, although it stands right in front of it. The relevant rule that is violated here concerns the existence of an entity revealing another type of shadow, except the perceived one. The position of the incongruent entity is meant here to suggest the powerful influence of shadow over light. On a conceptual level, the shadow of doubt, as well as the shadow of guilt are intended to cover and submit the light of innocence. As it grows bigger, the indexical shadow becomes capable to spread its features over the whole space.

From a more general perspective, the engagement of this type of shadow proposes incongruities that are more context-dependent than the other types of shadow. The depiction of shadow functions as the index that aims to signal that the other entity is revealing an incongruity. That is why, in order to highlight

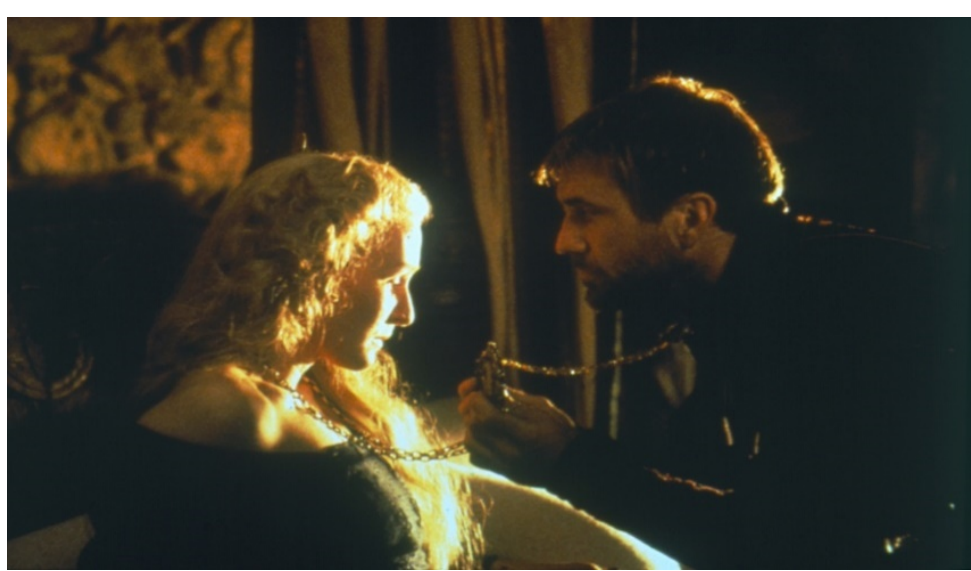

such incongruity, it is absolutely necessary to relate the shadow to the light source. By creating a balance between shadow and light, one configuring the other, the resolution assigned by the incongruities exploited by the indexical shadow may

cast a template framework.

All three types of shadow, revealing, transitional and indexical, are meant to exhibit an incongruity between the entities involved in a cinematic frame. Processed according to the relevance principle, the incongruity is intended to communicate the artistic and aesthetic intentions, as well as to suggest further meanings hidden within the frame. The first type of shadow is always involved in representing incongruities that include a revelatory aspect, while the second type of shadow stands for those incongruities which evoke a transitional aspect. The topic part is usually assumed by the casting entity for incongruities generated by the use of revealing shadow but, for those incongruities that are provided by the engagement of the transitional shadow, the topic itself becomes the factor causing the transition. For the third type of shadow, the indexical one, 
the employment of shadow sheds light on specific aspects that pass from one entity to the other.

There are similarities, as well as differences among the three types of shadow. Regarding the number of entities involved in the representation of the incongruity, the first and second type of shadow need two entities, but the third type needs only one. The ontology of the entities is different in the case of the first type of shadow, similar in the case of the second type but absent in the case of the third type of shadow. If for the first two types of shadow the locus of incongruity is given by the caster, for the third type it is the constituent. This fact shows that the topic is represented by the caster for the first type of shadow, while for the second type the topic causes the transition and for the third type the topic owns the property that is to be shared by the other entity. Thus, the resolution assumed by the first type of shadow is definitely revelation while transition represents the resolution for the second type. For the third type of shadow the resolution is supported by the property of the constituent.

The use of these types of shadow is destined to help the viewer decode the meaning, most of times, hidden within the frames they watch and it also prefigures further meanings suggested by the interactions between the entities included in such frames. Although the meaning construction is also verbally represented, the cinematic perspective adds new possibilities of building up further significations.

\section{Conclusion}

Engaging the three types of shadow in a cinematic representation aims not only at providing new possibilities for re-thinking the metaphor of shadow, but also at proposing new instruments of analysis. The depictions of shadow, in each case discussed here, share a property common to other rhetorical figures, either literary or cinematographic: deviation. By employing such deviant depictions, the natural and unambiguous perception of a cinematic frame or scene was prevented. Instead, governed by the principle of relevance, the shadow depictions generate further possibilities of building up new conceptual representations as well as interpretations. Due to the violation of the basic shadow perception rules, the possibility emerges for proper incongruities between the caster and its shadow, as well as between the caster and the light source depicting a shadow.

All three types of shadow are involved in generating specific incongruities. 
Be it that the incongruity focuses on the relation between caster and shadow representing distinct entities or on the same entity, but in different stages of evolution, or the incongruity is signalled by the shadow depiction functioning as an index, the use of shadow as metaphor, when transposed from philosophy to literature and cinematography, adapts its means of representations to reveal further significances.

\section{Works Cited}

Alberti, Leon Battista. On Painting, trans. Rocco Sinisgalli, Cambridge, Cambridge University Press, 2011.

Casati, R. The Shadow Club, New York, Knopf, 2003.

Dee, H. M., \& Santos, P. E.: "The Perception and Content of Cast Shadows: An Interdisciplinary Review" in Spatial Cognition \& Computation, No. 3, , 2011: 226-53.

Forceville, C. J. Pictorial Metaphor in Advertising, London and New York, Routledge, 1996.

Heidegger, Martin. The Essence of Truth. On Plato's Cave Allegory and Theaetetus, trans. T. Sadler, London and New York, Continuum, 2002.

Lakoff, G., \& Johnson, M., Metaphors We Live By, Chicago IL.,Chicago University Press, 1980.

Plato. Republic, trans. R. Waterfield, Oxford and New York, Oxford University Press, 1993.

Peirce, C. S. The Essential Peirce Volume 2, ed. N. Hauser \& Chr. Kloesel , Bloomington, Indiana University Press, 1998.

Shakespeare, William. The Norton Shakespeare, ed. Stephen Greenblatt, Walter Cohen, Jean E. Howard and Katharine Eisaman Maus, 2nd ed., Norton, 2008.

Stoichiță, Victor, Ieronim. A Short History of the Shadow, London, Reaktion Books, 1997.

Walton. K., L. "Pictures and Make-Believe" in Philosophical Review, No. 82, 283319, 1973.

Wollen, P. Signs and Meaning in the Cinema, London, 1998.

\section{Online Resources}

Figure 1: The revealing shadow

https://www.google.com/search?q=zeffirelli $\% 27 \mathrm{~s} \% 20$ hamlet $\% 20$ images\&tbm=isc 
h\&hl=ro\&tbs=rimg:CVpLPRHDLBTIYSsfwreYmwl3sgIGCgIIABAA\&sa=X\&ved= 0CB8QuIIBahcKEwiQpr2BzJzzAhUAAAAAHQAAAAAQEg\&biw $=1349 \& b i h=62$ 5\#imgrc=OFToGSCi5KYVSM\&imgdii=DpRkItiaD8K87M

Figure 2: The transitional shadow

https://www.google.com/search?q=zeffirelli\%27s+hamlet+images+claudius\&tbm =isch\&ved=2ahUKEwij38_R9qbzAhVIgaQKHSNcDoUQ2-

cCegQIABAA\&oq=zeffirelli\%27s+hamlet+images+claudius\&gs_lcp=CgNpbWcQ A1CKrA5Ys8EOYJXIDmgAcAB4AIABhgGIAY8IkgEDMC45mAEAoAEBqgELZ3 dzLXdpei1pbWfAAQE\&sclient=img\&ei=JMxVYaO9DsiCkgWjuLmoCA\&bih=571 \&biw $=1017 \&$ hl=ro\#imgrc=lsjdJhAQ5Oa-GM

Figure 3: The indexical shadow

https://www.google.com/search?q=zeffirelli\%27s\%20hamlet\%20images\&tbm=isc h\&hl=ro\&tbs=rimg:CVpLPRHDLBTlYSsfwreYmwl3sgIGCgIIABAA\&sa=X\&ved= 0CB8QuIIBahcKEwiQpr2BzJzzAhUAAAAAHQAAAAAQEg\&biw=1349\&bih=62 $5 \#$ imgrc $=8$ MsykW4Hlq078M 